\title{
Contributions of the chick embryo and experimental embryology to understanding the cellular mechanisms of neurulation
}

\author{
GARY C. SCHOENWOLF* \\ Department of Neurobiology and Anatomy, University of Utah School of Medicine, Salt Lake City, Utah, USA
}

\begin{abstract}
The chick embryo has served as a workhorse for experimental embryological studies designed to elucidate mechanisms underlying neurulation, the process that forms the neural tube, the rudiment of the entire adult central nervous system. Early chick embryos developing in whole-embryo culture can be readily manipulated in cut-and-paste-type experiments, and this attribute makes this model system unparalleled for studying the morphogenesis of embryos and their organ rudiments. How the chick embryo and experimental embryology have contributed to our understanding of critical events of neurulation are summarized.
\end{abstract}

KEY WORDS: cell-shape, convergent-extension, ectoderm, microfilament, microtubule, neural plate, oriented mitosis

\section{Introduction}

Experimental embryology has played an integral role in elucidating the cellular mechanisms that shape tissues during embryogenesis. Historically, a progression occurred in the use of vertebrate animal models for experimental embryology, generally moving from lower to higher vertebrates. Techniques for experimental manipulation were first developed using amphibian embryos, which offer several advantages such as easy and efficient lab-based animal husbandry of adults, year-round availability of embryos, large numbers of embryos with each spawning, rapid development of embryos, and development outside of a uterus in simple salt solutions. Experimental embryological techniques were soon adapted for use in chick embryos, and, eventually but to a far more limited extent, for use in fish and mouse embryos.

Beginning in the early $19^{\text {th }}$ century, the concept of Entwicklungsmechanismen, or developmental mechanisms, was introduced. To elucidate developmental mechanisms, such as the processes that underlie induction of the lens of the eye, amphibian embryos were treated experimentally, typically microsurgically, to elucidate the cause of developmental events. For example, an organ rudiment might be ablated to ask whether its presence is required for the development of a neighboring tissue. Or an organ rudiment might be transplanted to an ectopic location in an embryo to ask whether its presence is sufficient to alter the developmental course of another tissue in that area, inducing it to form something other than what it would normally form. Such experiments constitute what is typically called cut-and-paste experimental embryology. Thus, the field of embryology/developmental biology gradually transformed from solely descriptive studies based on observations of the development of embryos and their organ rudiments, usually studied microscopically with serial paraffin sections of fixed embryos, to analytical, dynamic studies on living embryos to determine the cellular mechanisms underlying morphogenesis. Similar approaches in which molecules are ablated (i.e., knocked down or knocked out) or over expressed grew out of cut-and-paste experimental embryology and are revealing the molecular and genetic mechanisms underlying morphogenesis.

The focus of this article is on the contributions of the chick embryo and experimental embryology to understanding the process of neurulation, that is, the cellular mechanisms that establish and convert a specialized region of ectoderm into a hollow, craniocaudally-elongated rudiment called the neural tube. The neural tube, along with the heart tube, is one of the first organs to appear in the embryo; it gives rise to the entire central nervous system of the adult. Although the use of amphibian, zebrafish, and mouse embryos has provided important information on how neurulation occurs, the chick embryo has served as a major workhorse for these studies. Essential attributes for this role include many of those listed above for amphibian embryos. Importantly, chick embryos can be removed from the yolk and cultivated in vitro from stages preceding neurulation (i.e., gastrulation) to well after completion of this process, and chick embryos develop as fundamentally flat, two-dimensional structures, greatly facilitating

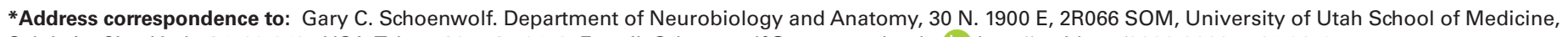
Salt Lake City, Utah, 84132-3401 USA. Tel: +1-801-581-6453. E-mail: Schoenwolf@ neuro.utah.edu (iD http://orcid.org/0000-0003-1764-2619

Supplementary Material (time lapse video) for this paper is available at: http://dx.doi.org/10.1387/ijdb.170288gs

Submitted: 31 October, 2017; Accepted: 10 November, 2017.

ISSN: Online 1696-3547, Print 0214-6282

(C) 2018 UPV/EHU Press

Printed in Spain 
the timelapse recording of neurulation (Supp. Fig. 1). Moreover, the use of the chick embryo might offer particular insight into human neurulation because both embryos begin their development as flat blastoderms, as opposed to the mouse embryo, for example, which consists of a cuplike blastoderm during neurulation.

\section{Overview of neurulation}

Fig. 1 illustrates the stages of chick neurulation, which are also shown in a more dynamic way in a timelapse video (Supp. Fig. 1). Neurulation starts as the embryo is undergoing gastrulation and its beginning is heralded by the thickening of the ectoderm surrounding Hensen's node, the avian equivalent of the Spemann organizer of amphibians. As this thickening occurs, the centralmost ectoderm becomes structurally and visibly (due to differences in optical density owing to its thickening) demarcated from the more peripheral ectoderm, with the former becoming the neural plate and the latter becoming the surface ectoderm. Cellsspanning the interface between these two ectodermal areas ultimately contribute to the neural crest and to cranial placodes, such as the nasal, lens, and otic placodes, as well as to the neurogenic (e.g., trigeminal) placodes.

Thickening of the ectoderm, or formation of the neural plate, is the first stage of neurulation. It results from the process of neural induction in which molecules secreted from the organizer act on the ectoderm to alter its fate. Neural induction has been studied in many vertebrate embryos, including the chick embryo.

Shortly after the neural plate forms it undergoes a dramatic change in its overall shape and begins to roll up. The nascent neural plate when viewed from its surface is an oval-shaped structure. During the second stage of neurulation, called shaping of the neural plate, the width (i.e., transverse dimension) of the neural plate narrows and its length (craniocaudal dimension) concomitantly increases. As shaping of the neural plate is underway, the third stage of neurulation, called bending of the neural plate, is initiated. During this process, the ectoderm spanning the lateral edges of the neural plate kinks to form structures called the neural folds, and these folds soon rise up (elevate) and begin to move toward one another (converge), establishing a transient, craniocaually elongated structure called the neural groove.

During the final stage of neurulation, called closure of the neural groove, the neural folds are brought into apposition in the dorsal midline and fuse with one another to establish the roof of a closed neural tube, which is simultaneously covered with a sheet of continuous surface ectoderm. Formation of the neural crest is intimately associated with both formation of the neural folds and closure of the neural groove. In the chick, bending of the neural plate and closure of the neural groove begin at the future midbrain level of the embryo and progress in a zippering-like fashion, both cranially to form the forebrain, and caudally to form the hindbrain and spinal cord.

It is important to emphasize that stages of neurulation overlap one another. For example, bending of the neural plate begins in the future midbrain region as shaping of the neural plate is occurring more caudally (Fig. 2A). Thus, there is a roughly cranial-to-caudal spatial progression in the stages of neurulation. In addition, there is a temporal progression at each craniocaudal level. The spatial progression can best be seen in whole embryos (Fig. 2A), and the temporal progression can best be seen in transverse views of the same level at progressively later stages (Fig. 2 B-D).

\section{Contributions of chick experimental embryology to our understanding of neurulation}

Of the four stages of neurulation, discussed above, the use of chick experimental embryology has provided the most insight into the understanding of two stages: shaping of the neural plate, and

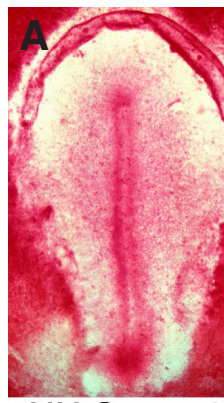

HH Stage 4
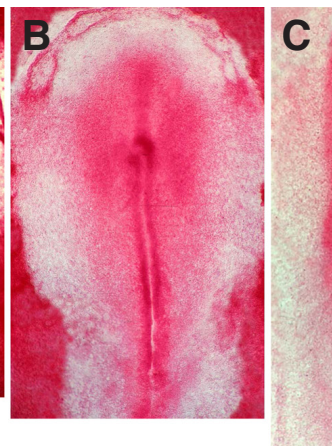

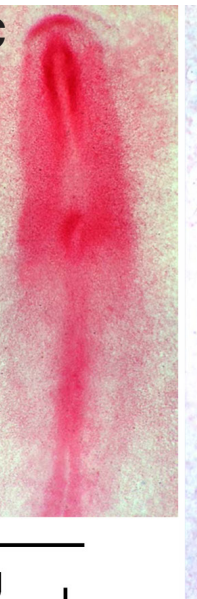

Shaping
Fig. 1. Selected images of fixed and stained chick embryos beginning at HH stage 4 (A) (flat neural plate stage) and ending at HH stage 11 (E) (closed neural tube stage), showing shaping $(\mathbf{B}, \mathbf{C})$ and bending of the neural plate $(\mathbf{C}, \mathbf{D})$, and closure of the neural groove (D,E).

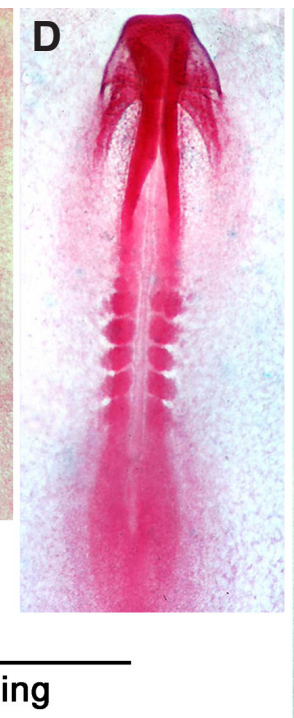

Bending

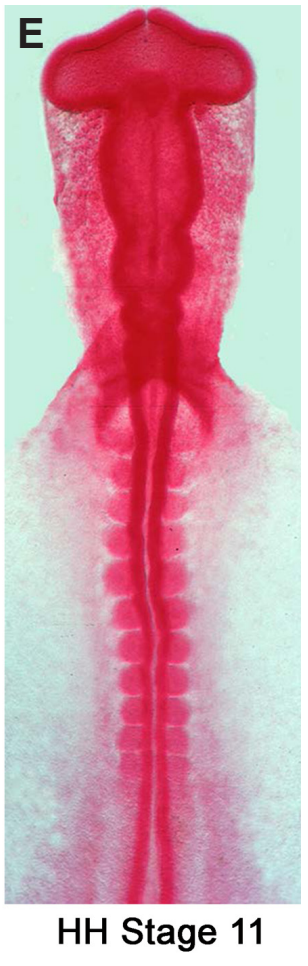

Closure 
bending of the neural plate to form the neural groove. After first providing a brief discussion of what I will refer to as the classical view of neurulation, a view based largely on the study of amphibian embryos, I will provide an overview of the chick experiments that provided new insight into neurulation and have resulted in the current view, a view generally considered to encompass both mouse and human neurulation, as well as chick neurulation. As discussed below, a major difference in neurulation between so-called lower vertebrates (reptiles and amphibians) and higher vertebrates (birds and mammals) is that extensive growth (i.e., mitosis) occurs during neurulation in the latter but not in the former.

\section{Classical view of neurulation}

In the 1970s, experiments designed to understand the cellular mechanisms of neurulation were first begun in earnest by pioneers in the field such as Antone Jacobson, Perry Karfunkel, and Tom Schroeder. Their studies, conducted primarily on amphibianembryos, led to a widely-accepted model, herein called the classical model, for explaining the cellular mechanisms driving shaping and bending of the neural plate. This model was based on three premises for shaping of the neural plate. First, that thickening of the neural plate is the result of apicobasal cell elongation rather than epithelial stratification (i.e., formation of a multilayered epithelium). A large number of histological and other types of studies have shown that the neural plate during neurulation consists of a pseudostratified, columnar epithelium, that is, an epithelium that is one-cell thick but contains multiple nuclei arrayed across its apicobasal dimension, giving the impression in histological sections that it consists of multiple cell layers. The second premise is that apicobasal cell elongation is driven by microtubules. This idea was based on studies that showed that neural plate cells, when viewed with transmission electron microscopy, contained extensive paraxial microtubules (i.e., microtubules aligned parallel to their elongating axis), and that depolymerizing microtubules with various agents blocked thickening of the neural plate. The third premise is that coordinated narrowing of the neural plate and craniocaudal lengthening is the result of convergent-extension driven by cell rearrangement. Convergentextension was first clearly described by Vogt (Vogt, 1929) and the role of cell rearrangement-namely, cell-cell intercalation-has been extensively studied during gastrulation of amphibian embryos, particularly in the morphogenesis of the early mesoderm, notably by Ray Keller and co-workers. Innovative computer modeling studies by Antone Jacobson and Richard Gordon (Jacobson and Gordon, 1976) suggested that the latter premise could be valid for shaping of the amphibian neural plate.

The classical model for bending of the neural plate is based on two premises. First, that bending involves changes in cell shape in the neural plate such that cells convert from columnar to wedge-or bottleshaped, a process referred to as cell wedging. This idea was based largely on a logical argument that cell wedging in an epithelial sheet would lead to bending, rather than on detailed observation of neural plate cells, which were assumed to undergo wedging throughout the bending neural plate. The second premise is that cell wedging is the result of the constriction of apical circumferential bands of microfilaments. This idea was based on studies that showed that neural plate cells, when viewed with transmission electron microscopy, contained apical circumferential bands of microfilament arranged in a purse-string-like fashion, and that depolymerizing microfilaments with various agents blocked bending of the neural plate.

Integrating the premises for shaping and bending of the neural plate into a comprehensive model yields the following three components of the classical model for neurulation: 1.) Changes in neural plate cell shape mediate shaping and bending of the neural plate. 2.) Changes in cell shape is driven by their cytoskeleton, namely, paraxial microtubules and apical circumferential bands of microfilaments. 3.) By extension, forces for neurulation are intrinsic, that is, all forces necessary for shaping and bending of the neural plate arise solely within the neural plate through the coordinated action of the cytoskeleton. Thus, according to the classical model, neurulation is an organ-rudiment-autonomous morphogenetic event.

\section{New insight into shaping and bending of the neural plate, based on chick experimental embryology}

\section{Shaping of the neural plate}

To determine the cellular mechanisms underlying shaping of the neural plate, it was first necessary to determine the changes in the overall size of the neural plate that occurred from the time
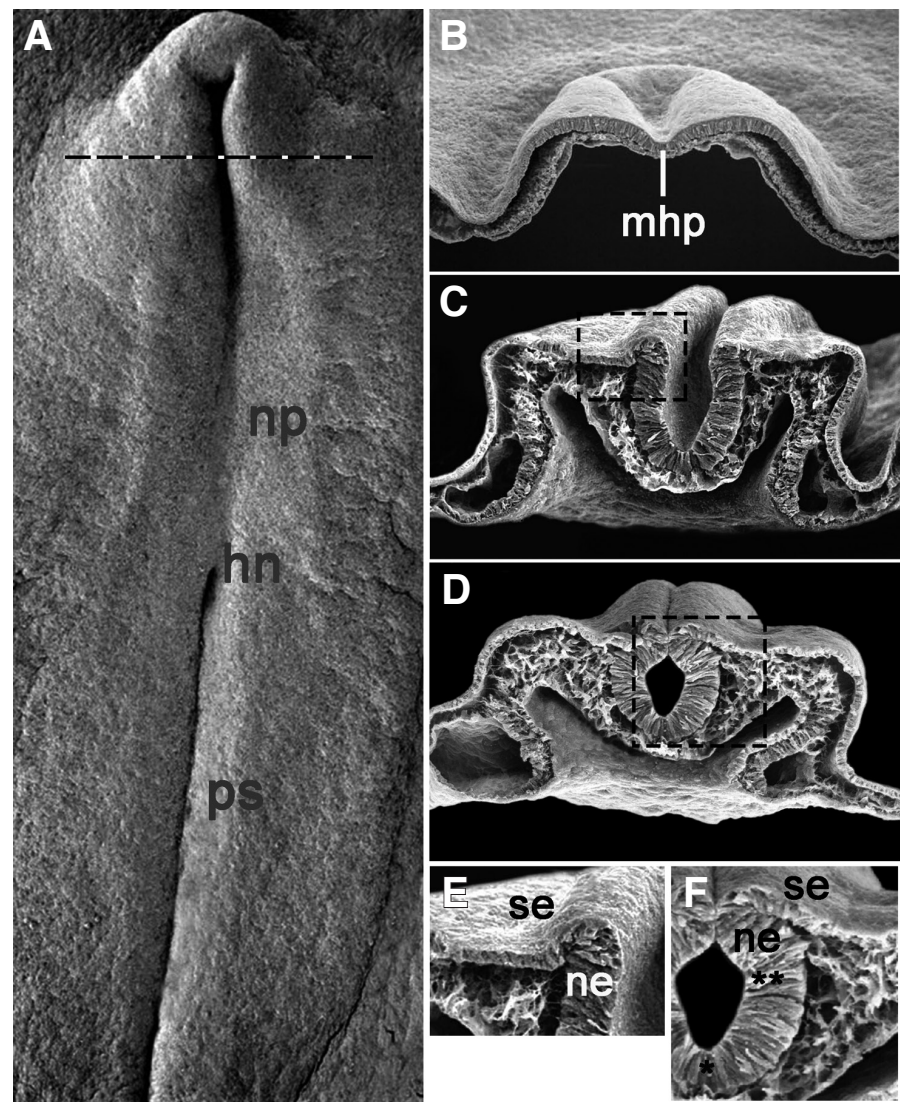

Fig. 2. Chick neurulation as viewed with scanning electron microscopy. (A) Intact embryo at HH stage 7 viewed from its dorsal surface. Shown are the neural plate (np), primitive streak (ps), and Hensen's node (hn). The dashed line indicates the level of the transverse fracture shown (B). (B-D) Transverse fractures through the future midbrain region at about $\mathrm{HH}$ stages 7 (B), 8- (C) and 8 (D). Dashed box in (C) is enlarged in (E). Dashed box in (D) is enlarged in (F). se, surface ectodermal layer of neural fold; ne, neuroepithelial layer of neural fold; single asterisk, median hinge point; double asterisks, dorsolateral hinge points. 
of its formation (Hamburger and Hamilton, 1951) $([\mathrm{HH}]$, Stage 4) to the time of completion of neural groove closure (HH Stage 11), a length of time extending approximately 24 hours. Using morphometric approaches, it was estimated that during neurulation the neural plate increased its overall volume by about 5 -fold, its thickness by 1.3-fold (i.e., by $30 \%$ ), and its length by almost 9 -fold; in addition, the width of the neural plate was halved (Schoenwolf, 1985). As in amphibians, it is believed that little or no growth occurs in individual cells of the ectoderm during these stages, but unlike at earlier stages when cleavage is occurring, daughter cells do seem to increase their volume after division to approximate that of the parental cell. Moreover, extensive extracellular matrix deposition does not seem to occur within the epithelium during neurulation. Thus, the most obvious explanation of the increase in volume is cell mitosis, with an estimated two to three divisions occurring during the approximate 24 hours of neurulation. Cell cycle studies support this contention, as the average cell cycle length in the neural plate during neurulation ranged from about 8 hours for lateral neural plate cells to about 12 hours for the most medial neural plate cells overlying the notochord (Smith and Schoenwolf, 1987). Interestingly, similar differences were shown in cell cycle lengths between lateral and medial neural plate cells in the mouse, but the length of the cell cycle in these regions is approximately half that occurring in the chick (i.e., 4 and 7 hours, respectively), suggesting that more cell divisions occur in the neural plate during neurulation in the mouse than in the chick (McShane et al., 2015).

The increase in thickness of the neural plate is the result of the apicobasal elongation of neural plate cells, as the neural plate (and, subsequently, the wall of the newly formed neural tube) in the chick embryo also consists of a pseudostratified, columnar epithelium, as described above in the classical model based on the amphibian embryo. Thus, the first premise of the classical model for neural plate shaping (that neural plate thickening is the result of apicobasal cell elongation) is also valid for the chick embryo. Cytokinesis is restricted to the apical side of the epithelium and daughter cells quickly extend to span the entire apicobasal extent of the epithelium. Thus, cell nuclei occupy multiple apicobasal positions within the epithelium, and as they progress through the cell cycle, they undergo interkinetic nuclear migration, that is, nuclei move apically as they replicate their DNA, undergo cytokinesis at the apex of the epithelium, and their daughter cell nuclei migrate basally as they immediately reenter the cell cycle, with a negligible Go/G1 period. Similarly, in the mouse neural plate, cell nuclei occupy multiple apicobasal positions within the epithelium and interkinetic nuclear migration occurs (Nikolopoulou et al., 2017).

It is not completely clear whether cell elongation is driven solely by microtubules in neural plate cells, as other potential mechanisms, such as changes in lateral cell adhesion have not been thoroughly studied. However, cooling embryos to $4^{\circ} \mathrm{C}$ after the neural plate cells have elongated (or treating embryos with chemicals that disrupt microtubules, such as colchicine or nocodazole), a process that depolymerizes microtubules, decreases cell heights by about $25 \%$ (Schoenwolf and Powers, 1987), suggesting that microtubules are the main driver of cell elongation during shaping of the neural plate. Similarly, cell elongation during neural keel formation in the zebrafish depends heavily on microtubules (Araya et al., 2016; Minc et al., 2009). Thus, the second premise of the classical model for neural plate shaping (that apicobasal cell elongation during neural plate formation is driven at least in large part by microtubules) is also valid for both chick and zebrafish embryos.

Experimental embryological studies in the chick embryo have been especially useful in elucidating the cellular mechanisms underlying coordinated mediolateral narrowing and craniocaudal lengthening of the neural plate during shaping. As discussed above, chick embryos are particularly amenable to cut-and-pastetype experiments. Moreover, these experiments can be done by transplanting donor cells that can be readily distinguished from host cells when developing in the host environment. One way to do this is to create quail-chick transplantation chimeras in which donor quail cells are transplanted in place of host chick cells and subsequently detected at the end of the experiment by using a quail-specific nuclear antibody, or before the availability of this antibody, Feulgen staining, which highlights differences in the nuclei of chick and quail cells. In one set of experiments, circular tissue plugs were "punched" from the neural plate using a fine micropipette, and plugs were then fixed, embedded in paraffin, and serially sectioned (Schoenwolf and Alvarez, 1989). The average number of cells spanning the diameter of the plug, as well as the total number of cells in the plug, were then calculated. Using micropipettes of the same diameter, plugs were removed from the neural plate of chick embryos developing in whole-embryo culture and replaced with plugs removed from the comparable area of the neural plate of quail embryos at the same stage. Host embryos were returned to the incubator for an additional 24 hours of development, allowing plugs to heal in place and the embryo to complete neurulation. Then, embryos were serially sectioned and processed for Feulgen staining to detect quail cells. The shape of the donor (quail) grafts in the host (chick) neural tube were reconstructed and measured, and the total number of cells in the graft, as well as the number of cells spanning the mediolateral width of the graft at each craniocaudal section level, were then calculated. This approach revealed that during the course of neurulation, grafts underwent three rounds of craniocaudal extension, with each round defined as a doubling of the graft's length; thus, during the course of neurulation, the graft increased its length 8-fold, closely approximating the amount of craniocaudal extension the neural plate normally undergoes during neurulation (Schoenwolf, 1985). Additionally, analyzing changes in cell number revealed that cells in the graft underwent 2-3 cell divisions (i.e., cell number increased 2- to 3-fold), consistent with the values obtained from cell-cycle studies (Smith and Schoenwolf, 1987). Finally, the number of cells spanning the midcraniocaudal extent of grafts was inversely correlated with the length of the graft. Thus, short grafts, which underwent little craniocaudal extension, had an increased number of cells spanning their midcraniocaudal extent (by about $1 / 3$ ), whereas long grafts, which underwent almost full craniocaudal extension, had about $1 / 6$ to $1 / 3$ the number of cells spanning their midcraniocaudal extent. This latter finding provided the first direct evidence that narrowing of the chick neural plate involved cell-cell intercalation, resulting in neural plate lengthening (i.e., convergent-extension). In addition, modeling studies suggested that oriented cell division also played a role in neural plate shaping, especially neural plate lengthening, with the prediction that the majority of mitotic figures would be oriented in the craniocaudal plane. Actual quantification of mitotic spindle orientation in the neural plate during its shaping (and bending) supported this suggestion (on average, about $54 \%$ of the spindles were oriented in the craniocaudal plane to place daughter cells into the elongating neural plate, and about $35 \%$ were oriented in 
the mediolateral plane (Sausedo et al., 1997). Thus, shaping of the chick neural plate involves three main cell behaviors: apicobasal cell elongation, cell-cell intercalation, and oriented mitosis. As for the first and second premises of the classical model for neural plate shaping, the third premise that coordinated narrowing of the neural plate and craniocaudal lengthening is the result of convergent-extension driven by cell rearrangement is true, but in addition, oriented cell division likely plays an important additional role in higher vertebrates. Oriented cell division similarly plays and important role in driving elongation of the neuroepithelium during zebrafish neurulation (Ciruna et al., 2006; Picone et al., 2010).

Experiments in which the surface ectoderm was removed from neurulating embryos in culture, isolating the neural plate, showed that the neural plate undergoes essentially normal shaping in isolation, providing strong support for the idea that shaping of the neural plate involves intrinsic forces generated solely by neural plate cells (Moury and Schoenwolf, 1995, Schoenwolf, 1988). However, the potential role in this process of underlying mesodermal cells (and perhaps endodermal cells), which undergo similar convergentextension movements, has not been rigorously explored. As inferred from the classical model for neurulation, the idea that shaping of the neural plate is largely an organ-autonomous event holds for shaping of the chick neural plate as well.

Recent studies, based largely on mouse mutants, have revealed that convergent extension during shaping of the neural plate is regulated through the planar cell polarity $(\mathrm{PCP})$ pathway (reviewed by Nikolopoulou et al., 2017; also see Ciruna et al., 2006; Curtin et al., 2003; Wallingford and Harland, 2002), consistent with both cell intercalation being the motor for convergent extension in the neural plate and the process being an organ-autonomous event. In fact, convergent extension is greatly inhibited in PCP mutant mice, resulting in open neural tubes that have greater widths and shorter lengths than those of controls (Ybot-Gonzalez et al., 2007).

\section{Bending of the neural plate}

To determine the cellular mechanisms underlying bending of the neural plate, a series of experiments was required. The first premise of the classical model for bending - that cell wedging causes neural plate bending - was tested by asking whether widespread cell wedging occurred within the neural plate during its bending. By comparing nuclear position within the apicobasal dimension of the neuroepithelium from histological sections to scanning electron micrographs of transverse fractures of the neuroepithelium it was determined that nuclei position was a reliable indicator of overall cell shape in the neural plate (Schoenwolf and Franks, 1984). Specifically, four cell shapes were identified: wedge-shaped (or flask-shaped) cells (containing basally positioned nuclei), inverted wedge-shaped cells (containing apically positioned nuclei), spindleshaped cells (containing nuclei positioned in the wall of the epithelium at least one nuclear diameter from both the apical or basal sides of the epithelium), and globular cells, containing mitotic figures positioned at the apical side of the epithelium. Prior to bending and at all stages of bending, only a minority of neuroepithelial cells (25$30 \%$ ) are wedge-shaped; most are spindle-shaped (60-70\%). With formation of the notochord, cells in the midline of the neural plate decreased their heights and the percentage of wedge-shaped cells in this localized region increased to about $50 \%$. Because subsequently the neural folds formed and rotated (i.e., elevated) around this midline axis, forming the neural groove, the midline area was named the median hinge point (Fig. 2B). With further bending, the percentage of wedge-shaped cells in this area increased to about $70 \%$, while in the more lateral neural plate, the overall percentage of wedge-shaped cells increased by less than $10 \%$ during bending. Cutting-and-pasting-type experiments revealed that this difference in the shapes of median and lateral neuroepithelial cells is induced by the notochord (Smith and Schoenwolf, 1989). As the neural folds elevated in the future brain region, but not in the future spinal cord region, two dorsolateral hinge points formed as the surface ectoderm folded against the neuroepithelium to deepen the neural folds (Fig. 2 C-F). Within these hinge points, the percentage of cell wedging also increased (to about $60 \%$ ) as the neural folds underwent convergence. Thus, in contrast to the first premise of the classical model for neural plate bending, cell wedging is not occurring throughout the neuroepithelium but is instead restricted to localized regions called hinge points. Similar hinge points and localized areas of cell wedging also form in the mouse neural plate (McShane et al., 2015; Nikolopoulou et al., 2017).

The second premise of the classical model for bending of the neural plate-that apical circumferential bands of microfilaments drive cell wedging during bending - was tested by treating embryos with cytochalasin to depolymerize microfilaments, and then again quantifying neuroepithelial cell shapes (Schoenwolf et al., 1988). Such treatment did not prevent neural plate shaping, median neural plate furrowing, median cell wedging, or elevation of the neural folds, demonstrating that microfilaments do not drive these events. However, dorsolateral neural plate furrowing, dorsolateral cell wedging, and convergence of the neural folds were frequently inhibited, suggesting a role for apical microfilaments in these events. Formation of the median hinge point in the mouse neural plate, a process that involves localized cell wedging as in the chick neural plate, can also occur in an actin microfilament-independent manner (Ybot-Gonzalez and Copp, 1999).

The fact that cell wedging can still occur in the chick median hinge point after depolymerization of microfilaments raises two questions: how does cell wedging occur, and does cell wedging truly involve apical constriction, the first premise of the classical model for bending of the neural plate? An alternative way for cell wedging to occur is through basal expansion. In scanning electron micrographs of fractures through the neuroepithelium, apexes of most cells are very narrow regardless of the amount of neural plate bending or the mediolateral location of the cells in the neuroepithelium. Thus, it is hard to imagine how further constriction of the apex could occur or that such further constriction would have a major impact on bending of the neural plate. As discussed above, because the apicobasal position of the nucleus of neuroepithelial cells necessarily defines their shape (i.e., the diameter of the portion of the cell containing the nucleus is several-fold greater that the cell's apical or basal extension) and interkinetic nuclear migration occurs, one way to cause cell wedging over time would be to increase the length of the cell cycle during the phase when the nucleus resides basally. Detailed cell-cycle analyses supported this possibility, suggesting that regulation of the cell cycle causes basal expansion and consequently cell wedging in the bending neural plate (Smith and Schoenwolf, 1988). Thus, the first premise of the classical model for neural plate bending (that cells undergo cell wedging solely as a result of apical constriction), as well as the second premise of this model (that cell wedging is the result of the constriction of apical circumferential bands of microfila- 
ments), may hold for only some of the wedging cells of the neural plate (e.g., in the dorsolateral hinge points), but likely not for the majority of those cells.

Microsurgical isolation experiments were used to ask to what extent bending of the neural plate is independent of surrounding tissues (Moury and Schoenwolf, 1995, Schoenwolf, 1988, Smith and Schoenwolf, 1991). In other words, is bending of the neural plate driven by intrinsic biomechanical forces, extrinsic biomechanical forces, or a combination of both types of forces? Formation of the median hinge point, including cell wedging and epithelial furrowing, still occurred after isolation of the median strip of neural plate from the paired lateral regions of neural plate, suggesting that formation of the median hinge point is autonomous to midline tissues and does not require interactions or biomechanical forces from surrounding tissues. Similarly, separation of the lateral neural plate from more lateral tissues (i.e., surface ectoderm and its underlying mesoderm and endoderm) did not prevent median hinge point formation. However, the neural plate failed to roll up into a neural groove; thus, only a flat, open "neural tube" formed. This result suggests that neuroepithelial cell wedging by itself is insufficient to cause neural fold elevation and convergence, and that lateral tissues play a role in neural plate bending. However, it still remains unclear exactly what that role is. In conclusion, as inferred from the classical model for neurulation, the idea that bending of the neural plate is largely an organ-autonomous event does not hold for bending of the chick neural plate.

Lateral tissues could play two potential roles in bending of the neural plate and closure of the neural groove. First, lateral tissues might transmitsignals to the lateral neuroepithelium that differentially regulate cell behaviors in this region, possibly causing formation of the dorsolateral hinge points and convergence of the neural folds to close the neural groove. Second, lateral tissues might provide biomechanical, pushing forces. Of course, these potential roles are not mutually exclusive. Although the former possibility still remains because it has not been directly tested, microsurgical experiments suggest that lateral tissues provide forces for bending of the neural plate and for closure of the neural groove. In a set of experiments described above in which the median neural plate was separated bilaterally from the lateral neural plate, median cell wedging and apical furrowing still occurred but a "mini" neural tube did not form, as the neuroepithelium remained flattened, rather than rounding up. But what happened to the lateral neural plate? In these, the surface ectoderm extensively expanded medially and each "half" neural plate independently from the other underwent elevation and convergence toward the dorsal midline. However, convergence did not stop there: the plate continued to rotate as much as an additional $180^{\circ}$; (see especially Fig. 11e in Smith and Schoenwolf, 1991). Thus, surface ectoderm has the power to displace and rotate the neuroepithelium, but it is not known if such forces are sufficient to drive bending or are only commensurate with neural fold displacement via independent mechanisms.

Forces generated by lateral tissues could originate in the surface ectoderm, mesoderm, and/or endoderm. It seems likely that all three of these tissues generate forces, as all three tissues expand toward the midline during neurulation, as well as during other events temporally and spatially coupled with neurulation, such as lateral body folding, which contributes to the formation of the heart tube and underlying foregut, ventral folding events not unlike that occurring dorsally during neurulation. Microsurgical experiments again in the chick embryo have revealed that removal of both the lateral endoderm and mesoderm together has essentially no effect on bending of the neural plate, provided that the surface ectoderm is left intact (Alvarez and Schoenwolf, 1992). However, ablation of only the surface ectoderm, leaving the lateral endoderm and mesoderm intact, blocks bending (Hackett et al., 1997). Thus, the surface ectoderm is the most critical lateral tissue required for neurulation. How might the surface ectoderm generate forces to push the neural folds dorsomedially during their elevation and convergence? Analyses of medial tissue expansion in surface ectodermal isolates, quail/chick transplantation chimeras, and mitotic spindle orientations in the surface ectoderm, suggest that surface ectodermal expansion generates medially-directed forces and that these arise from changes in surface ectodermal cell behaviors that are similar to those occurring in the neural plate during its shaping (Moury and Schoenwolf, 1995, Sausedo et al., 1997, Schoenwolf and Alvarez, 1991), namely, cell-cell intercalation and oriented cell division, but the main direction of surface ectodermal expansion as a result of these cell behaviors is mediocaudal rather than strictly caudal, as in the neural plate.

The surface ectoderm is also likely to be important for neural plate bending and neural groove closure because it partners with the lateral neuroepithelium to form the neural folds. Neural folds clearly play an essential role in these processes and they likely provide forces for the last bit of convergence, bringing the neural folds into midline apposition as each layer of the fold expands medially along their common interface (Fig. 2 C-F) during the final stages of neural groove closure (Lawson and Schoenwolf, 2001).

\section{Conclusions and future directions}

\section{The cooperative model of neurulation}

Experimental embryological studies primarily in the chick embryo have provided new insight into the cellular mechanisms of neurulation that lead to the current model for shaping and bending of the neural plate. In this model, it is proposed that shaping of the neural plate is largely an autonomous event, not requiring lateral tissues, and that it results from changes in the behaviors of neuroepithelial cells, namely, apicobasal cell elongation, cell-cell intercalation, and oriented cell division. Bending of the neural plate, however, requires the cooperation of lateral tissues, especially the surface ectoderm, which undergoes medial expansion resulting from changes in the behaviors of its cells, namely, cell-cell intercalation and oriented cell division. Finally, it is proposed that the neural fold interface plays an important role in the final events of neural groove closure by providing a substrate for tissue expansion. Thus, neurulation is the result of cellular events that occur both intrinsic to the neuroepithelium as well as extrinsic to it in lateral tissues.

Many questions remain about how neurulation occurs. In particular, we know little about how the last stage of neurulation occurs, closure of the neural groove and fusion of the neural folds. Also, how cellular behaviors generate forces for tissue morphogenesis is largely unknown; new innovative approaches will be needed to tackle this difficult problem. Finally, how cellular behaviors are coordinated across multiple tissues in time and space needs to be resolved, as well as deciphering the molecular mechanisms that regulate changes in fundamental cell behaviors driving morphogenesis. Its seems for at least the foreseeable future that use of the chick embryo and experimental embryological approaches 
will continue to provide an advantageous approach for answering these and other important questions in the field.

\section{Acknowledgments}

I wish to thank the many students, postdocs, and visiting faculty members who have contributed enormously to the chick studies on neurulation in my lab. It has been one of the great joys of my life to work closely with such dedicated, enthusiastic, intelligent, and creative people. Our research for these studies was supported by grants mainly from the National Institutes of Health, for which I am extremely grateful.

\section{References}

ALVAREZ, I.S. and SCHOENWOLF, G.C. (1992). Expansion of surface epithelium provides the major extrinsic force for bending of the neural plate. J. Exp. Zool. 261: 340-348.

ARAYA, C., WARD, L.C., GIRDLER, G.C. and MIRANDA, M. (2016). Coordinating cell and tissue behavior during zebrafish neural tube morphogenesis. Dev Dyn 245: 197-208.

CIRUNA, B., JENNY, A., LEE, D., MLODZIK, M. and SCHIER, A.F. (2006). Planar cell polarity signalling couples cell division and morphogenesis during neurulation. Nature 439: 220-224.

CURTIN, J.A., QUINT, E., TSIPOURI, V., ARKELL, R.M., CATTANACH, B., COPP, A.J., HENDERSON, D.J., SPURR, N., STANIER, P., FISHER, E.M., NOLAN, P. M., STEEL, K. P., BROWN, S. D., GRAY, I. C. and MURDOCH, J. N. (2003). Mutation of Celsr1 disrupts planar polarity of inner ear hair cells and causes severe neural tube defects in the mouse. Curr Biol 13: 1129-1133.

HACKETT, D.A., SMITH, J.L. and SCHOENWOLF, G.C. (1997). Epidermal ectoderm is required for full elevation and for convergence during bending of the avian neural plate. Dev. Dynamics 210: 1-11.

HAMBURGER, V. and HAMILTON, H.L. (1951). A series of normal stages in the development of the chick embryo. J. Morph. 88: 49-92.

JACOBSON, A.G. and GORDON, R. (1976). Changes in the shape of the developing vertebrate nervous system analyzed experimentally, mathematically and by computer simulation. J Exp Zool 197: 191-246.

LAWSON, A. and SCHOENWOLF, G.C. (2001). New insights into critical events of avian gastrulation. Anat. Rec. 262: 238-252.

McSHANE, S.G., MOLE, M.A., SAVERY, D., GREENE, N.D., TAM, P.P. and COPP, A.J. (2015). Cellular basis of neuroepithelial bending during mouse spinal neural tube closure. Dev Biol 404: 113-124.

MINC, N., BRATMAN, S.V., BASU, R. and CHANG, F. (2009). Establishing new sites of polarization by microtubules. Curr Biol 19: 83-94.

MOURY, J.D. and SCHOENWOLF, G.C. (1995). Cooperative model of epithelial shaping and bending during avian neurulation: autonomous movements of the neural plate, autonomous movements of the epidermis, and interactions in the neural plate/epidermis transition zone. Dev. Dynamics 204: 323-337.
NikOlopoulou, E., GAleA, G.L., ROLO, A., GREENE, N.D. and COPP, A.J. (2017). Neural tube closure: cellular, molecular and biomechanical mechanisms. Development 144: 552-566.

PICONE, R., REN, X., IVANOVITCH, K.D., CLARKE, J.D., MCKENDRY, R.A. and BAUM, B. (2010). A polarised population of dynamic microtubules mediates homeostatic length control in animal cells. PLoS Biol 8: e1000542.

SAUSEDO, R.A., SMITH, J.L. and SCHOENWOLF, G.C. (1997). Role of nonrandomly oriented cell division in shaping and bending of the neural plate. J. Comp. Neurol. 381: 473-488.

SCHOENWOLF, G.C. (1985). Shaping and bending of the avian neuroepithelium: Morphometric analyses. Dev. Biol. 109: 127-139.

SCHOENWOLF, G.C. (1988). Microsurgical analyses of avian neurulation: Separation of medial and lateral tissues. J. Comp. Neurol. 276: 498-507.

SCHOENWOLF, G.C. and ALVAREZ, I.S. (1989). Roles of neuroepithelial cell rearrangement and division in shaping of the avian neural plate. Development 106: 427-439.

SCHOENWOLF, G.C. and ALVAREZ, I.S. (1991). Specification of neurepithelium and surface epithelium in avian transplantation chimeras. Development 112: 713-722.

SCHOENWOLF, G.C., FOLSOM, D. and MOE, A. (1988). A reexamination of the role of microfilaments in neurulation in the chick embryo. Anat. Rec. 220: 87-102.

SCHOENWOLF, G.C. and FRANKS, M.V. (1984). Quantitative analyses of changes in cell shapes during bending of the avian neural plate. Dev. Biol. 105: 257-272.

SCHOENWOLF, G.C. and POWERS, M.L. (1987). Shaping of the chick neuroepithelium during primary and secondary neurulation: Role of cell elongation. Anat. Rec. 218: 182-195.

SMITH, J.L. and SCHOENWOLF, G.C. (1987). Cell cycle and neuroepithelial cell shape during bending of the chick neural plate. Anat. Rec. 218: 196-206.

SMITH, J.L. and SCHOENWOLF, G.C. (1988). Role of cell-cycle in regulating neuroepithelial cell shape during bending of the chick neural plate. Cell Tiss. Res. 252: 491-500.

SMITH, J.L. and SCHOENWOLF, G.C. (1989). Notochordal induction of cell wedging in the chick neural plate and its role in neural tube formation. J. Exp. Zool. 250: 49-62.

SMITH, J.L. and SCHOENWOLF, G.C. (1991). Further evidence of extrinsic forces in bending of the neural plate. J. Comp. Neurol. 307: 225-236.

VOGT, W. (1929). Gestaltungsanalyse am Amphibienkeim mit örtlicher Vitalfärbung. II. Teil. Gastrulation und Mesodermbildung bei Urodelen und Anuren. Wilhelm Roux's Arch. Dev. Biol. 120: 384-706.

WALLINGFORD, J.B. and HARLAND, R.M. (2002). Neural tube closure requires Dishevelled-dependent convergent extension of the midline. Development 129: 5815-25.

YBOT-GONZALEZ, P. and COPP, A.J. (1999). Bending of the neural plate during mouse spinal neurulation is independent of actin microfilaments. Dev Dyn 215: 273-283.

YBOT-GONZALEZ, P., SAVERY, D., GERRELLI, D., SIGNORE, M., MITCHELL, C.E., FAUX, C.H., GREENE, N.D. and COPP, A.J. (2007). Convergent extension, planar-cell-polarity signalling and initiation of mouse neural tube closure. Development 134: 789-799. 


\section{Further Related Reading, published previously in the Int. J. Dev. Biol.}

Neural crest ontogeny during secondary neurulation: a gene expression pattern study in the chick embryo

Liliana Osório, Marie-Aimée Teillet, Isabel Palmeirim and Martin Catala

Int. J. Dev. Biol. (2009) 53: 641-648

https://doi.org/10.1387/ijdb.072517lo

\section{Early neurogenesis in Amniote vertebrates}

N M Le Douarin

Int. J. Dev. Biol. (2001) 45: 373-378

http://www.intjdevbiol.com/web/paper/11291868

Neurulation in amniote vertebrates: a novel view deduced from the use of quail-chick chimeras

N M Le Douarin, M A Teillet and M Catala

Int. J. Dev. Biol. (1998) 42: 909-916

http://www.intjdevbiol.com/web/paper/9853821

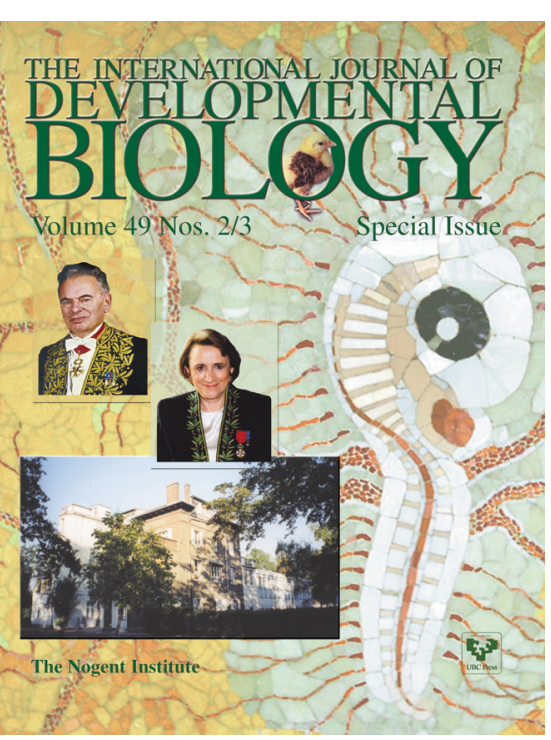

5 yr ISI Impact Factor $(2016)=2.421$
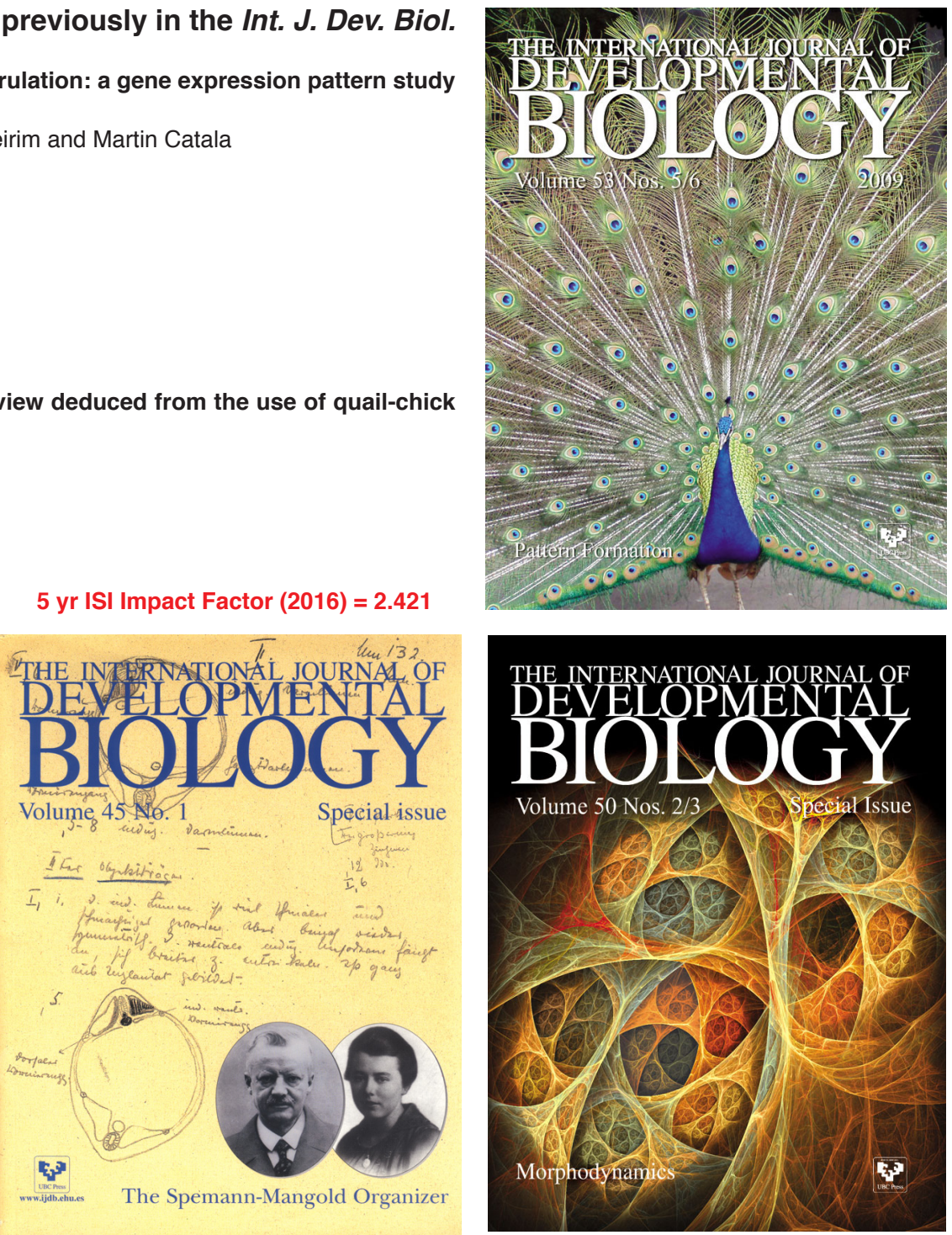